\title{
Deletion of the HAMP domains from the histidine kinase CaNik1p of Candida albicans or treatment with fungicides activates the MAP kinase Hog1p in S. cerevisiae transformants
}

\author{
Mohammed El-Mowafy ${ }^{1,2}$, Mahmoud M Bahgat ${ }^{3,4}$ and Ursula Bilitewski ${ }^{*}$
}

\begin{abstract}
Background: Microorganisms use two-component signal transduction (TCST) systems to regulate the response of the organism to changes of environmental conditions. Such systems are absent from mammalian cells and are thus of interest as drug targets. Fungal TCST systems are usually composed of a hybrid histidine kinase, comprising the histidine kinase (HisKA) domain and a receiver domain, a histidine phosphotransfer protein and a response regulator. Among the 11 groups of fungal histidine kinases, group III histidine kinases are of particular relevance as they are essential for the activity of different groups of fungicides. A characteristic feature is the N-terminal amino acid repeat domain comprising multiple HAMP domains, of which the function is still largely unknown. In Candida albicans, a fungal human pathogen, three histidine kinases were identified, of which CaNik1p is a group III histidine kinase. Heterologous expression of this protein in Sacchromyces cerevisiae conferred susceptibility to different fungicides. Fungicide activity was associated with phosphorylation of the mitogen activated protein kinase $\operatorname{Hog} 1 \mathrm{p}$.

Results: We have constructed mutated versions of CaNik1p, from which either all HAMP domains were deleted (CaNik1p $\triangle$ HAMP) or in which the histidine kinase or the receiver domains were not-functional. Expression of CaNIK1 $\triangle H A M P$ in S. cerevisiae led to severe growth inhibition. Normal growth could be restored by either replacing the phosphate-accepting histidine residue in CaNik1p $\triangle$ HAMP or by expressing CaNIK1 $\triangle H A M P$ in S. cerevisiae mutants, in which single genes encoding several components of the HOG pathway were deleted. Expression of proteins with non-functional histidine kinase or receiver domains resulted in complete loss of susceptibility to antifungals, such as fludioxonil. Conditions leading to growth inhibition of transformants also led to phosphorylation of the MAP kinase Hog1p.

Conclusion: Our results show that functional histidine kinase and receiver domains of CaNik1p were essential for antifungal susceptibility and for activation of the Hog1p. Moreover, for the first time we show that deletion of all HAMP domains from CaNik1p led to activation of Hog1p without an external stimulus. This phenotype was similar to the effects obtained upon treatment with fungicides, as in both cases growth inhibition correlated with Hog $1 p$ activation and was dependent on the functionality of the conserved phosphate-accepting histidine residue.
\end{abstract}

Keywords: Group III histidine kinase, CaNik1p, Point mutations, Protein domains, Fludioxonil, Transformed Saccharomyces cerevisiae, HAMP domains

\footnotetext{
* Correspondence: ursula.bilitewski@helmholtz-hzi.de

${ }^{1}$ AG Biological Systems Analysis, Helmholtz Centre for Infection Research

(HZI), Inhoffenstr. 7, 38124 Braunschweig, Germany

Full list of author information is available at the end of the article
} 


\section{Background}

Immune-compromised patients are at high risk of becoming infected by opportunistic fungi, such as Candida and Aspergillus sp. Candida sp. are the fourth most frequent cause of hospital acquired blood stream infections and up to $90 \%$ of HIV patients receive mucosal candidiasis at least once [1]. Although infections with non-albicans Candida $s p$. have emerged in recent years [2], the species $C$. albicans is still responsible for the majority of the cases [3,4].

Several antifungals are available in the market, yet, toxicity and/or development of resistance represent major concerns [5]. Among these is the former "gold standard" therapeutic amphotericin B that invariably causes toxicity in patients, negating the importance of its fungicidal activity. Although azoles and echinocandins represent the most widely used treatments of candidiasis, the acquisition of resistance can occur, leading to the risk of recurrent infections [6,7]. Thus antifungals which impact new targets and have minimal side effects are urgently needed [7].

In fungi, two-component signal transduction (TCST) systems have been implicated in osmotic and oxidative stress responses, cell-cycle control, red/far-red light responses, and virulence switches from non-pathogenic to pathogenic states [8-10]. Since TCST systems are absent in mammalian cells, they are attractive targets for the development of new antifungals with probably minimal side effects in humans [7].

Typical TCST systems in fungi include a histidine kinase (HK), a histidine phosphotransfer protein (HPT) and a response regulator protein (RR). The best understood fungal TCST system is part of the High Osmolarity Glycerol (HOG) pathway in S. cerevisiae. In the absence of osmotic stress, the transmembrane HK ScSln1p is active. This HK activity leads to phosphorylation of a histidine residue in the catalytic domain, the so-called HisKA domain, from which the phosphate group is transferred to an aspartic acid residue in an internal receiver domain (REC). Therefore, these HKs are called hybrid HKs. The phosphate group is then shuttled through the HPT protein Ypd1p to the terminal RR proteins Skn7p and Ssk1p [8,11]. Phosphorylated Skn7p is a direct regulator of gene expression, whereas phosphorylated Ssk1p is not able to activate downstream targets. Under conditions of high osmolarity, ScSln1p is inactive, resulting in the dephosphorylation of both RRs. Unphosphorylated Skn7p becomes inactive, whereas unphosphorylated Ssk1p activates a downstream mitogen-activated protein kinase (MAPK) module, in particular the MAP3K Ssk2p resulting in phosphorylation of the MAPK Hog1p [7,12-15]. Phosphorylated Hog1p upregulates the transcription of genes, which encode enzymes that play a key role in glycerol production and maintenance of the intracellular water balance, allowing adaptation to high-osmolarity conditions [13]. Thus, the HK ScSln1p is a negative regulator of the
MAPK Hog1p. Likewise, disruption of ScSLN1 results in the accumulation of unphosphorylated Ssk1p without external stimulus and thus, constitutive activation of Hog1p, which is lethal [14].

While $S$. cerevisiae has a single HK, namely $\operatorname{ScSln} 1 \mathrm{p}, C$. albicans has three HKs: CaSln1p, CaNik1p (also called Cos1) and Chk1p [8]. CaNik1p is considered to be a cytosolic enzyme, as it lacks the hydrophobic amino acids indicative of membrane-spanning domains (Figure 1) [16]. The protein is not essential for survival, and a gene deletion mutant could be generated [16-18]. CaNik1p plays an important role in hyphal formation in C. albicans on solid media $[8,18]$. Additionally, the deletion mutant was found to be less virulent in a mouse model for systemic candidiasis [8]. According to the classification scheme of HKs [9], ScSln1p and CaSln1p are group VI HKs while CaNik1p is a group III HK.

Several chemical classes of fungicides, such as phenylpyrroles (fludioxonil), dicarboximides (iprodione) and polyketide secondary metabolites of ambruticins, exert their antifungal effects by activating the HOG signaling pathway, resulting in the accumulation of both glycerol and free fatty acids [19-22]. It is assumed that in the absence of high external osmolarity, artificial induction of excess intracellular glycerol causes leakage of cellular contents and ultimately results in cell death [21,22]. Mutations in group III HKs are frequently associated with fungicide resistance [19], showing the relevance of these enzymes for fungicide activity and placing also these HKs upstream the MAPK Hog1p. It is still discussed, whether group III HKs are negative (as is ScSln1p) [23] or positive [24] regulators of Hog1p. S. cerevisiae lacks group III HKs and is usually resistant to the fungicides mentioned above. However, fungicidal sensitivity is gained by heterologous functional expression of group III HKs in S. cerevisiae correlating with Hog1p phosphorylation [25-28].

All classes of HKs share the conserved phosphateaccepting domains HisKA, REC and an ATP-binding

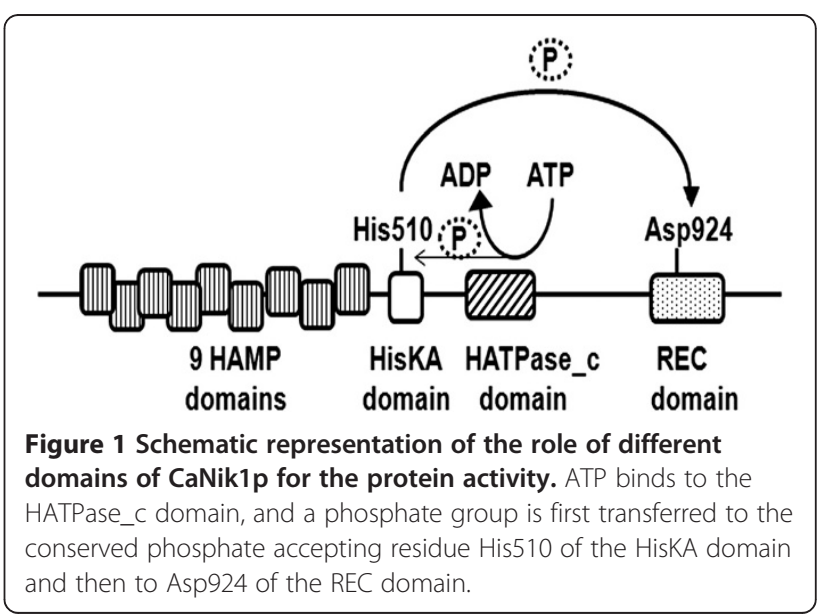


domain called HATPase_c domain. Based on homology with other HKs, the histidine residue 510 and the aspartate residue 924 in CaNik1p were identified as the essential phosphorylatable residues for the HisKA and the REC domains respectively (Figure 1) [17]. The ATP-binding domain comprises a characteristic N-box with two asparagine residues, which are N623 and N627 in CaNik1p [17]. The $\mathrm{N}$-box is known to be essential for ATP binding [29] and deletion of a single asparagine residue was associated with complete inhibition of ATP binding in the HK EnvZ [30].

Group III HKs are characterized by additional amino acid repeats in the $\mathrm{N}$-terminal part with a length of approximately 90 amino acids each. The repeats contain evolutionary conserved amino acid sequences called HAMP domains. Such abbreviation is due to the frequent occurrence of such domains in histidine kinases, adenylcyclases, methyl accepting chemotaxis proteins and phosphatases, which are proteins associated with signal transduction in both prokaryotic and lower eukaryotic organisms [31]. More than 26400 proteins with HAMP domains exist in the SMART data base. These domains were shown to play an active role in intramolecular signal transduction in prokaryotic sensor kinases. They are composed of about 50 amino acid residues each with two amphipathic helices [32-34] which probably rotate when the sensor domain of the protein is activated as recently elucidated from NMR analysis [35,36]. Unlike the bacterial HK, which usually possess a single HAMP domain, fungal group III HKs have several consecutive HAMP domains. In the five $\mathrm{N}$-terminal amino acid repeats of CaNik1p [16-18] we identified nine HAMP domains of a concatenated structure forming four pairs each with an overall length of 92 amino acids and a single HAMP domain in the remaining truncated amino acid repeat [25].

To study the role of the various protein domains in the function of group III HKs different protein mutants were constructed. In Hik1p, a group III HK from Magnaporthe grisea, phosphate acceptance on both the conserved histidine and aspartic acid residues in the catalytic and the receiver domains respectively was essential for the susceptibility to phenylpyrroles and ambruticin VS4 [26,27]. Deletions of single pairs of HAMP domains from the HK CaNik1p of $C$. albicans were associated with decreased susceptibility to fungicides, showing the relevance of these domains for fungicide activity [25] and deletion of four out of five amino acid repeats from the HK DhNik1p of Dabaryomyces hansenii generated a constitutively active HK, which was resistant to osmotic stress and fungicide treatment $[23,37]$.

As C. albicans is a human pathogen, understanding the relevance of the $\mathrm{N}$-terminal nine HAMP domains and of the HisKA, HATPase_c and REC domains of CaNik1p for the action of antifungal compounds can guide development of new antimycotic strategies. To achieve this goal, point mutations were introduced in the HisKA, HATPase_c and REC domains of CaNIK1 which should render these domains non-functional. In addition, a deletion mutant for all HAMP domains was generated. Due to the ease of genetic manipulation of $S$. cerevisiae the plasmids harboring the mutated CaNIK1 were used to transform $S$. cerevisiae followed by testing viability, sensitivity to fungicides and phosphorylation of the MAPK Hog1p upon fungicidal treatment.

\section{Methods}

\section{Organisms and growth conditions}

S. cerevisiae BWG1-7a [38] and BY4741 [39] were used in the present study (Table 1).

Prior to transformation, S. cerevisiae was grown in YPD medium (Sigma-Aldrich) at $30^{\circ} \mathrm{C}$. S. cerevisiae transformants were selected and maintained in SD-ura (according to [40]), at $30^{\circ} \mathrm{C}$. To obtain high cell density before induction of transgene expression, the transformants were cultivated at $30^{\circ} \mathrm{C}$ in SD-ura for $36 \mathrm{~h}$. To induce transgene expression from the $36 \mathrm{~h} \mathrm{SD}$-ura culture, an overnight culture, a preculture $(2-3 \mathrm{~h})$ and ultimately a working culture were prepared in SG-ura. For growth of the reference $S$. cerevisiae strain uracil was added at a concentration of $40 \mathrm{mg} / \mathrm{l}$.

Solidified media were prepared by addition of $1.5 \%$ bacto agar (Difco).

E. coli XL1-Blue growth, transformation and plasmid DNA preparation were performed using standard methods according to the manufacturer's instructions.

\section{Mutagenesis of the cloned CaNIK1 gene in the pYES2 plasmid and expression of the mutated constructs in $S$. Cerevisiae transformants:}

The plasmid pYES2-CaNIK1-TAG [25] was used as a template for all the generated mutants in the present work. It encodes the wild-type CaNik1p protein fused to a HIS/ FLAG tag at the C-terminus. Point mutations were introduced in the HisKA (H510Q), HATPase_c (N627D) and REC (D924N) domains using the quick-change sitedirected mutagenesis kit (Stratagene). The nucleotide sequences of the primers used, where the nucleotide changes were introduced to lead to the desired mutations, are given in Table 2 . The PCR reaction mixture, the amplification program, the digestion with the restriction enzyme DpnI (Stratagene) and the transformation of the competent cells were carried out according to the manufacturer's instructions.

In CaNik1p (1081 aa), all the HAMP domains (63-485 aa) were deleted using the in-fusion $\mathrm{HD}$ cloning kit (Clontech). Briefly, the in-fusion enzyme is able to fuse up to four DNA fragments with a linearized vector upon recognizing 15 bp overlapping sequences at their ends. 
Table $1 \mathrm{~S}$. cerevisiae strains used in this study

\begin{tabular}{|c|c|c|c|}
\hline Strain designation & Genotype & Transformed with & Reference \\
\hline BWG1-7a & Mat a ura3-52 leu2-3,112 his4-519 ade1-100 & - & {$[38]$} \\
\hline YES & BWG1-7a & pYES2 & This study \\
\hline NIK & BWG1-7a & pYES2-CaNIK1-TAG & [25] \\
\hline H510 & BWG1-7a & pYES2-CaNIK1(H510Q) & This study \\
\hline D924 & BWG1-7a & pYES2-CaNIK1(D924N) & This study \\
\hline N627 & BWG1-7a & pYES2-CaNIK1(N627D) & This study \\
\hline$\Delta \mathrm{Ha}$ & BWG1-7a & pYES2-CaNIK1 1 HAMP & This study \\
\hline$\Delta \mathrm{HaH} 510$ & BWG1-7a & pYES2-CaNIK1 1 HAMP(H510Q) & This study \\
\hline$\Delta \mathrm{H} 3 \mathrm{H} 4$ & BWG1-7a & pYES2-CaNIK1 $1224-315 \Delta 327-418 a a$ & [27] \\
\hline BY4741 & Mat a his $3 \Delta 1$; leu2 $\Delta 0$; met15 $\Delta 0$; ura3 $\Delta 0$ & - & [39] \\
\hline$\Delta \mathrm{Hb}$ & BY4741 & pYES2-CaNIK1 $\triangle H A M P$ & This study \\
\hline$\Delta \mathrm{HbH} 510$ & BY4741 & pYES2-CaNIK1 $\triangle H A M P(H 510 Q)$ & This study \\
\hline$\Delta$ ssk1 & BY4741, YLR006c::kanMX4 & - & [49] \\
\hline$\Delta \mathrm{pbs} 2$ & BY4741, YJL 128c::kanMX4 & - & [49] \\
\hline$\Delta$ hog & BY4741, YLR113w::kanMX4 & - & [49] \\
\hline$\Delta \mathrm{Hb} \Delta \mathrm{ssk} 1$ & $\Delta s s k 1$ & pYES2-CaNIK1 1 HAMP & This study \\
\hline$\Delta \mathrm{Hb} \Delta \mathrm{pbs} 2$ & $\Delta p b s 2$ & pYES2-CaNIK1 $\triangle H A M P$ & This study \\
\hline$\Delta \mathrm{Hb} \Delta$ hog & $\Delta h o g$ & pYES2-CaNIK1 $\triangle H A M P$ & This study \\
\hline
\end{tabular}

To allow this fusion, the 15 bp overlaps were introduced to the primers which were used to amplify the target fragments.

The pYES2 vector was linearized using the restriction enzyme HindIII and the pYES-CaNIK1-TAG vector was used as a template for amplification of the gene fragments. The sequence of CaNIK1 upstream of the fragment encoding the HAMP domains (1-186 bp) was amplified using the HMPF1 and HMPR1 primers (Table 2). HMPF1 included homologous $15 \mathrm{bp}$ with the end of the linearized vector downstream of the galactose promoter. The CaNIK1 fragment located downstream the sequence encoding the HAMP domains and extended by the His-FLAG tag
(1454-3243 bp) was amplified using the HMPF2 and HMPR2 primers (Table 2). HMPF2 and HMPR2 shared 15 bp homologous stretches with the 172-186 bp fragment of CaNIK1 and with the other end of the HindIIIlinearized pYES2 vector, respectively. HindIII restriction sites were introduced into the sequences of the HMPF1 and HMPR2 primers. After separation of the PCR amplified fragments by electrophoresis on 1.2\% agarose gels, the gel pieces carrying the amplification products were excised and the DNA was purified using a gel extraction kit (Qiagen). The purified fragments were ligated into the digested pYES2 vector using the in-fusion enzyme according to the manufacturer's instructions.

Table 2 Oligonucleotides used in this study

\begin{tabular}{|c|c|c|}
\hline Primer & Sequence $\left(5^{\prime}-3^{\prime}\right)$ & Used in mutation of: \\
\hline F2Gln & CTAGCGAACATGTCGCAAGAGATACGTACACC & HisKA domain \\
\hline R2Gln & GGTGTACGTATCTCTTGCGACATGTTCGCTAG & \\
\hline TAsnF & CTTAACTTGGCTGGTGATGCTATTAAGTTTAC & HATPase_c domain \\
\hline TAsnR & GTAAACTTAATAGCATCACCAGCCAAGTTAAG & \\
\hline AAsnF & GATGTGGTGTTGATGAATGTGCAAATGCCTGTAATG & REC domain \\
\hline AAsnR & CATTACAGGCATTTGCACATTCATCAACACCACATC & \\
\hline HMPF1 & AGGGAATATTAAGCTTATGAACCCCACTAAAAAACCACG & HAMP domains \\
\hline HMPR1 & GTTCGCGTTITGGATTITTCTAG & \\
\hline HMPF2 & TCCAAAAACGCGAACAGGAATACTGCGGCTAGAGAAGCTG & \\
\hline HMPR2 & GCTCGGTACCAAGCTTTCAGTGGTGATGGTGATGATGTCC & \\
\hline
\end{tabular}


The existence of the introduced mutations was further confirmed by sequencing the generated constructs (Dept. GNA, HZI, Braunschweig) using primers spanning the target fragments. The mutated constructs were used to transform S. cerevisiae using the lithium acetate method [40]. Transformants (Table 1) were selected on SD-ura agar plates.

\section{Susceptibility assays}

In 96 well microtiter plates, working cultures of the transformants were incubated in $180 \mu \mathrm{l}$ SG-ura supplemented with the appropriate concentrations of the antifungals in triplicates for $24 \mathrm{~h}$. The starting OD at $620 \mathrm{~nm}$ was 0.03 and measured using a $\mu$ Quant scanning microplate spectrophotometer (Biotek). To detect growth inhibitory effects, the $\mathrm{OD}_{620 \mathrm{~nm}}$ was again measured after $24 \mathrm{~h}$. The antifungals fludioxonil and iprodione were obtained from Fluka, whereas ambruticin VS3 was produced as described and kindly provided by K. Gerth and R. Jansen (HZI, Braunschweig) [41].

\section{Detection of Hog1p phosphorylation}

Phosphorylation of the MAPK Hog1 was investigated in transformants with CaNIK1 carrying point mutations as previously described [25]. Briefly, from precultures in SG-ura working cultures of the transformants were prepared in SG-ura containing $10 \mu \mathrm{g} / \mathrm{ml}$ fludioxonil with a starting $\mathrm{OD}_{620 \mathrm{~nm}}$ of 0.2 . Cells were harvested $15 \mathrm{~min}$ after the start of the working culture by centrifugation. Sorbitol (1 M) was used as a positive control, as it is known to stimulate phosphorylation of the MAPK Hog1p via the induction of osmotic stress [42].

To avoid Hog1 phosphorylation caused by cold stress [43], cells were directly shock frozen in liquid nitrogen after centrifugation. Frozen cell pellets were mechanically disrupted by grinding with the mini-dismembrator U (B. Braun Biotech, Melsungen, Germany) in the presence of lysis buffer (10 $\mathrm{mM}$ sodium phosphate buffer $\mathrm{pH}$ 7, supplemented with $5 \mathrm{mM} \mathrm{NaCl}, 5 \mathrm{mM} \mathrm{KCl}$, protease and phosphatase inhibitors (cOmplete ULTRA, mini, EDTA free and PhosSTOP (Roche)). Protein concentrations of the supernatants were determined using the BCA assay [44].

A total of $5 \mu \mathrm{g}$ protein per sample was separated by SDS-PAGE (12.5\%) and proteins were blotted onto a PVDF membrane. Phosphorylated Hog1 was detected using the combination of an anti-phospho p38 MAPK (Thr180/182) 3D7 rabbit monoclonal antibody (Cell Signaling Technology) with an HRP-conjugated antirabbit IgG (Cell Signaling Technology) as secondary antibody. Incubation of the antibodies was followed by the addition of a peroxidase-specific chemiluminescence substrate (ECL; Advance Western Blotting Detection Kit, GE Healthcare). The bound antibodies were removed by treatment with 1xRe-Blot Plus Solution (Millipore) and subsequently total Hog1p was detected using anti-Hog1 (y-215) sc-9079 rabbit polyclonal IgG (Santa Cruz Biotechnology) and the above mentioned secondary antibody followed by visualization with the ECL substrate.

\section{Detection of phosphorylation of Hog1p in S. cerevisiae transformed with CaNIK1pDHAMP}

Due to the growth inhibitory effect resulting from the expression of CaNik1p $\Delta$ HAMP in the $\Delta$ Ha strain, phosphorylation of Hog1p was investigated at an early time point after inducing the expression of CaNik1p $\triangle$ HAMP. Therefore $\Delta \mathrm{Ha}$ was first cultivated in SD-ura until $\mathrm{OD}_{620 \mathrm{~nm}}=1$. Cells were harvested by centrifugation and transferred to SG-ura (starting $\left.\mathrm{OD}_{620 \mathrm{~nm}}=0.2\right)$. After incubation at $30^{\circ} \mathrm{C}$ for 195 and $210 \mathrm{~min}$, samples were centrifuged and treated as previously mentioned for the detection of Hog1p phosphorylation by Western blotting. Fludioxonil was added as an inducer of Hog1 phosphorylation (positive control) after $180 \mathrm{~min}$ at a final concentration of $10 \mu \mathrm{g} / \mathrm{ml}$.

To confirm the expression of CaNik1p $\Delta$ HAMP, $\Delta \mathrm{Ha}$ cells were harvested after $180 \mathrm{~min}$ incubation in SG-ura, disrupted and the FLAG-tagged protein was detected using a HRP-conjugated monoclonal anti-FLAG antibody (Sigma-Aldrich).

\section{Results}

The conserved domains of CaNik1p were essential for the susceptibility of $S$. cerevisiae transformants to antifungals After alignment with other HKs, in CaNik1p histidine 510 and aspartate 924 were identified as the essential residues for the HisKA and the REC domains respectively [17] and asparagine 627 for the N-box of the ATP-binding domain. Hence, to inhibit the conserved phosphorylation reactions within CaNik1p, mutant genes were generated, in which either Asn627 from the HATPase_c domain was substituted by aspartate (N627D), His510 by glutamine (H510Q) or Asp924 by asparagine (D924N). S. cerevisiae was transformed with the plasmids carrying the mutated CaNIK1 genes, and the resultant transformants were treated with the antifungals fludioxonil, iprodione and ambruticin VS3. As shown in Figure 2, the strain YES transformed with the empty vector was resistant to all fungicides, while the strain NIK was susceptible to the studied antifungals. The $\mathrm{H} 510 \mathrm{Q}$ and $\mathrm{D} 924 \mathrm{~N}$ point mutations in the HisKA and REC domains respectively, led to complete loss of susceptibility, while the N627D substitution in the HATPase_c domain only decreased the susceptibility to the fungicides in comparison to the strain NIK.

His510 and Asp924 are the conserved phosphateaccepting residues in the HisKA and the REC domains, respectively, which are required for kinase function of hybrid HKs. They are phosphorylated by the histidine 

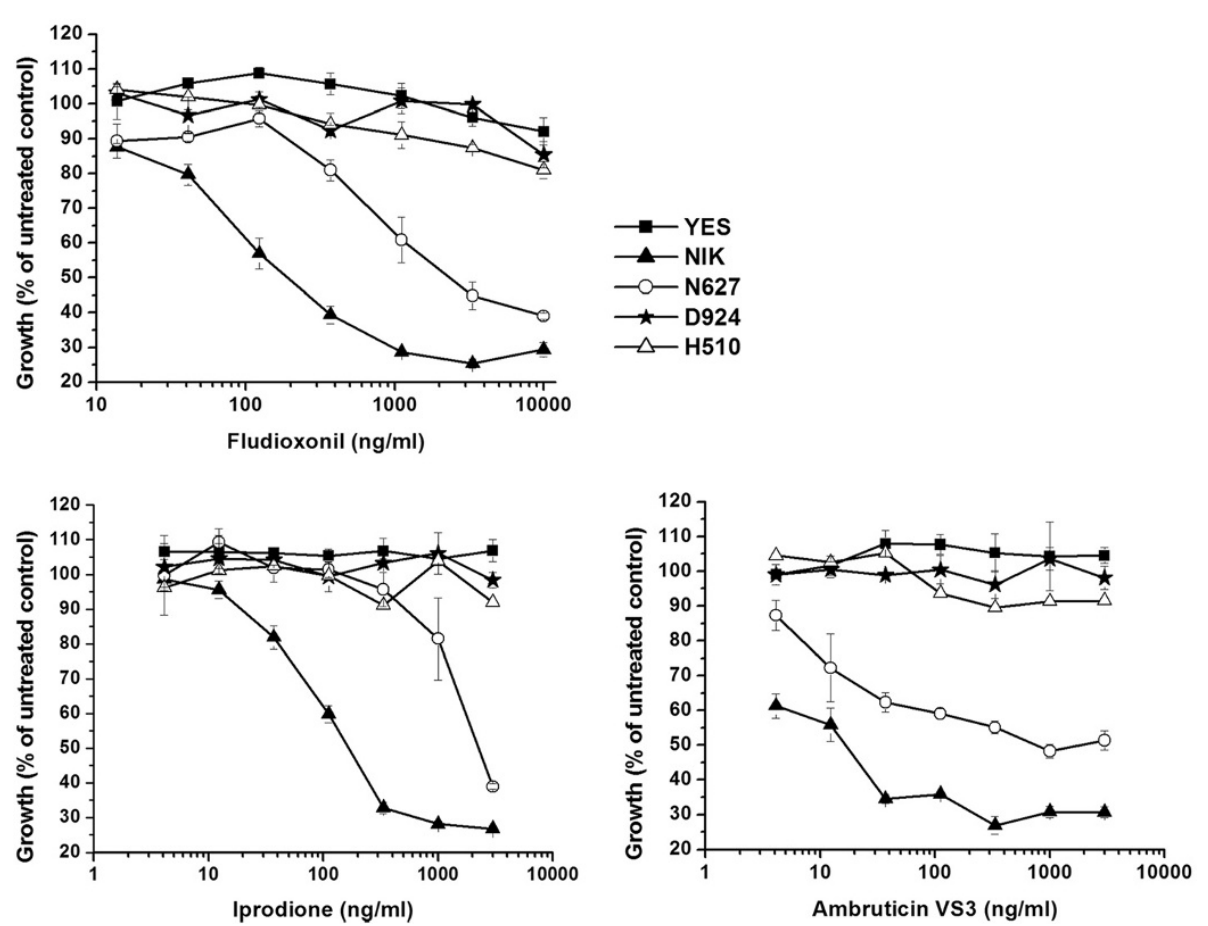

Figure 2 The conserved domains of CaNik1p were essential for the susceptibility to the fungicides. The phenylpyrrole fludioxonil, the dicarboximide iprodione and the myxobacterial secondary metabolite ambruticin VS3 were used as representative antifungal compounds targeting fungal group III histidine kinases. Error bars represent the standard deviation from three independent experiments.

kinase activity of the protein (His510) and the subsequent phosphate-transfer to the REC domain within the same protein (Asp924). Loss of fungicide susceptibility of the respective mutants suggested that the functionality of both the HisKA and the REC domain was essential for the antifungal activity.

Probably the N627D mutation did not completely prevent ATP binding to the HATPase_c domain and as a result only a partial effect was obtained.

\section{Functional HisKA, HATPase_c and REC domains were essential for the phosphorylation of Hog1p after fludioxonil treatment}

Treatment with fludioxonil led to phosphorylation of the MAPK Hog1p, i.e. to the activation of the HOG pathway, in S. cerevisiae transformed with full-length and truncated forms of CaNIK1 [25]. Therefore, phosphorylation of Hog1p was also analyzed after fludioxonil-treatment of $S$. cerevisiae transformed with CaNIK1 carrying the H510Q, N627D and D924N point mutations. Phosphorylation of the protein was totally abolished in the mutant strains D924 and H510, while phosphorylation of Hog1p in strain N627 was decreased but not totally inhibited (Figure 3). Thus, activation of Hog1p correlated with the inhibition of the yeast's growth by fludioxonil and both effects required the functionality of the domains that are essential for the histidine kinase function of the protein, which involves phosphorylation of both His510 and Asp924 of CaNik1p.

Since high concentrations of sorbitol activate the HOG pathway via inhibition of the HK $\operatorname{Sin} 1$, treatment of the transformants with $1 \mathrm{M}$ sorbitol was used as a positive control.

\section{Normal growth of the yeast was inhibited upon expression of CaNik1p $\triangle \mathrm{HAMP}$ and was restored by inhibition of the HOG pathway}

Previous work had shown that deletion of single and double pairs of HAMP domains of CaNik1p affected the susceptibility of the resultant mutants to the fungicides [25], and for the HK DhNik1 it was described that deletion of four out of five amino acid repeats generated a constitutively active HK, which could not be inhibited by fludioxonil [23]. Thus we decided to delete all HAMP domains from CaNIK1p. Transforming $S$. cerevisiae with a plasmid carrying a truncated version of CaNIK1, in which all HAMP domains were deleted from the protein, resulted in the $\Delta \mathrm{Ha}$ and $\Delta \mathrm{Hb}$ strains (Table 1 ). These strains were able to grow on SD-ura agar plates, where expression of CaNIK1 1 HAMP was not induced. Surprisingly no growth was observed on SG-ura plates, where galactose induced the expression of CaNIK1 $\triangle H A M P$ (Figure 4). This indicated that the presence of CaNIK1 1 HAMP had inhibitory effects on the growth of the $S$. cerevisiae transformant, 


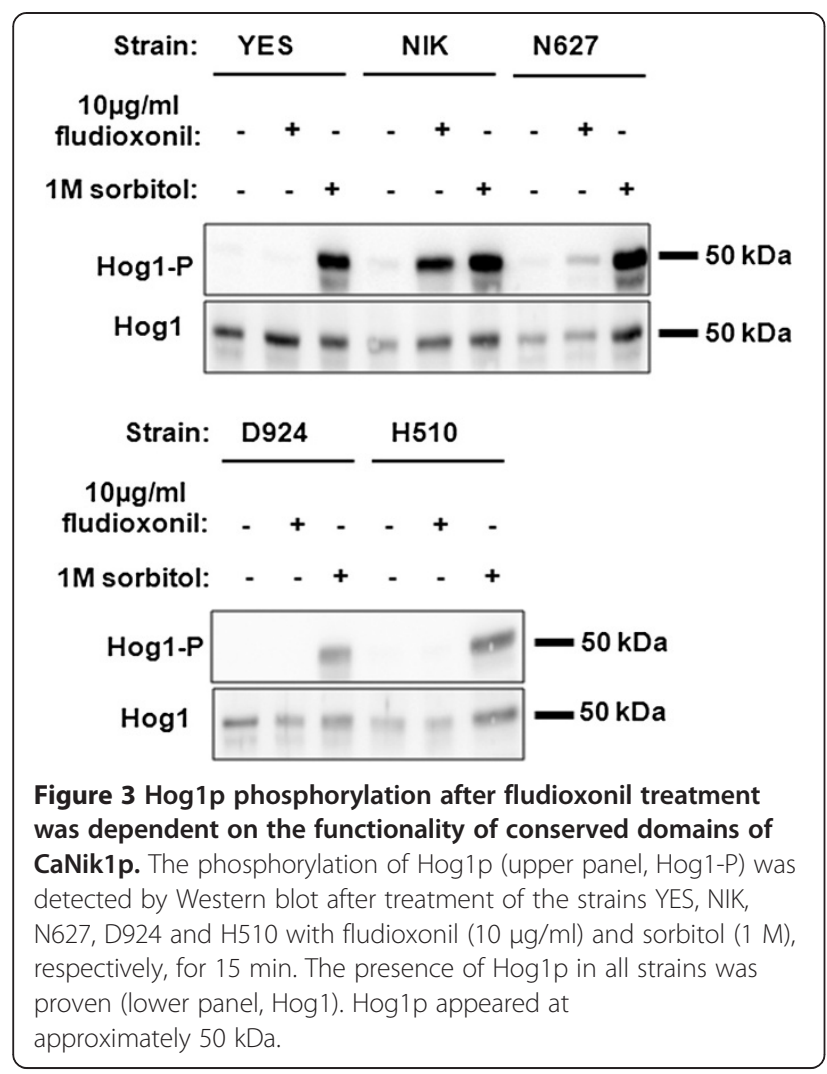

whereas deletion of up to two pairs of HAMP domains did not affect growth of the transformed strain $\triangle \mathrm{H} 3 \mathrm{H} 4$ [25] (Figure 4A). Simultaneous inactivation of the HisKA domain by the $\mathrm{H} 510 \mathrm{Q}$ point mutation restored normal growth of the resultant transformed strains $\Delta \mathrm{HaH} 510$ and $\triangle \mathrm{HbH} 510$ (Figure 4).

Limitation of Hog1p activity is essential for the survival of $S$. cerevisiae even under normal growth conditions, as a constitutively active MAP2K Pbs2p, which leads to constitutive activation of Hog1p, is toxic [45]. Thus, we assumed that constitutive activation of Hoglp could be the reason for the growth inhibitory phenotype resulting from the expression of CaNIK1 IHAMP. Therefore, S. cerevisiae strains with single gene deletions in the response regulator SSK1 (strain $\triangle$ ssk1) or components of the Hog1p MAPK module, namely the MAP2K PBS2 (strain $\triangle \mathrm{pbs} 2$ ) and the MAPK HOG1 (strain $\triangle \mathrm{hog}$ ), were transformed with the plasmid pYES2-CaNIK1 $1 H A M P$. These transformants showed normal growth on SG-ura plates (Figure 4B), proving that the growth inhibitory effect associated with the expression of CaNIK1 HHAMP was dependent on the functionality of the HOG pathway.

\section{Expression of CaNIK1 $\triangle H A M P$ resulted in constitutive} phosphorylation of Hog1p that was dependent on the conserved phosphate-accepting histidine residue To further analyze the involvement of Hog1p activity, the phosphorylation state of Hog1p was investigated. Due to the growth inhibitory effect resulting from the expression of CaNIK1 1 HAMP, the transformant strain $\triangle \mathrm{Ha}$ was first cultivated on the glucose-containing medium SD-ura that does not induce CaNIK1 $\triangle H A M P$ expression to produce sufficient biomass for protein analysis. Subsequently, the expression of CaNIK1 1 HAMP was induced by incubating the cells in the galactose-containing medium SG-ura. Gene

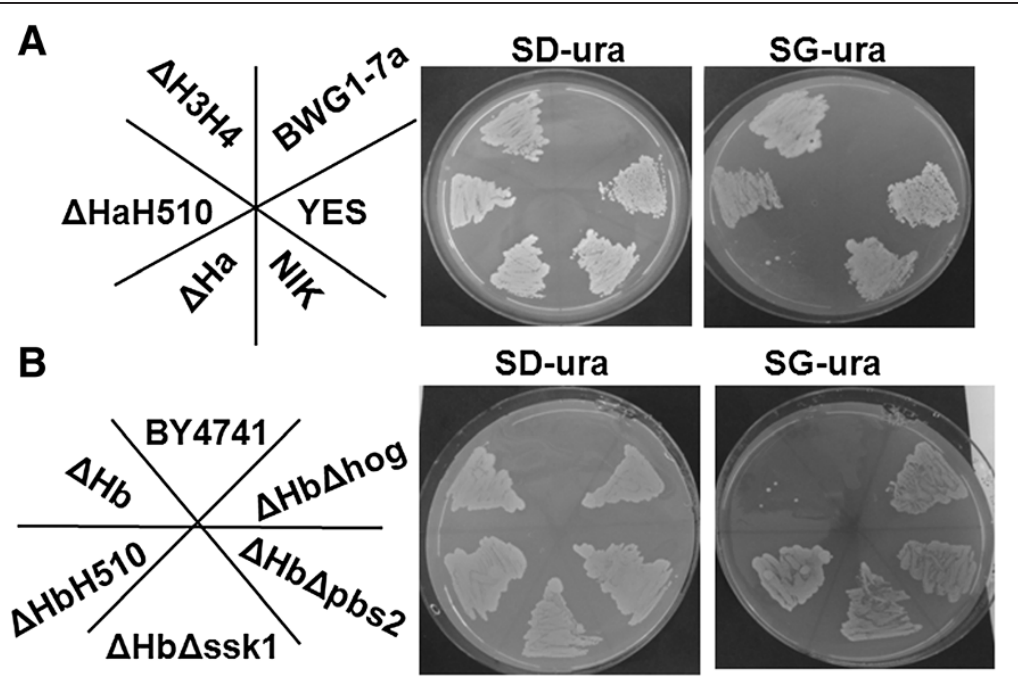

Figure 4 CaNIK1 $\triangle$ HAMP expression led to growth inhibition that was dependent on His510 (A) and a functional HOG pathway (B). (A) Strains BWG1-7a, YES, NIK, $\triangle \mathrm{Ha}, \Delta \mathrm{HaH} 510$ and $\Delta \mathrm{H} 3 \mathrm{H} 4$ were streaked on SD-ura and SG-ura agar plates and incubated at $30^{\circ} \mathrm{C}$ for 4 days. Strain BWG1-7a was the parent strain which is auxotrophic for uracil. (B) Strains BY4741, $\Delta \mathrm{Hb} \Delta$ hog, $\Delta \mathrm{Hb} \Delta \mathrm{pbs} 2, \Delta \mathrm{Hb} \Delta s \mathrm{sk} 1, \Delta \mathrm{HbH} 510$ and $\Delta \mathrm{Hb}$ were streaked on SD-ura and SG-ura agar plates and incubated at $30^{\circ} \mathrm{C}$ for 4 days. BY 4741 was the parent strain of the single gene deletion mutants, which is auxotrophic for uracil. SG-ura medium induced the expression of the transgenes via the galactose promoter. 
expression and protein synthesis were allowed for $180 \mathrm{~min}$ before fludioxonil was added. Presence of CaNik1p $\triangle$ HAMP was confirmed by Western blot using an anti-FLAG-antibody (see Additional file 1). Phosphorylation of Hog1p was examined after an additional 15 and $30 \mathrm{~min}$ (in total $195 \mathrm{~min}$ and $210 \mathrm{~min}$ respectively) (Figure 5). After fludioxonil treatment, phosphorylation of Hog1p was observed in the transformant strain NIK1 carrying the full-length protein, and in the transformant strain $\Delta \mathrm{Ha}$, whereas no phosphorylation was detected in the strains with the empty plasmid (YES) and with the additional H510Q mutation $(\Delta \mathrm{HaH} 510)$, respectively. Hog1p was phosphorylated in the transformant strain $\Delta \mathrm{Ha}$ even without the presence of fludioxonil, while such constitutive phosphorylation was not observed in the strains NIK and $\triangle \mathrm{HaH} 510$ (Figure 5). Thus, deletion of all HAMP domains from CaNik1p led to constitutive activation of Hog1p without any further external stimulus, which appears to be the reason for the growth inhibitory phenotype of the transformant strain $\Delta \mathrm{Ha}$ in galactose-containing medium.

\section{Discussion}

We previously showed that expression of the group III HK from the human fungal pathogen C. albicans, CaNIK1 in S. cerevisiae resulted in susceptibility of the transformants to the fungicides fludioxonil, iprodione and ambruticin VS3 [25]. Moreover, the fungicidal activity was decreased by deletion of single or double pairs of the $\mathrm{N}$-terminal HAMP domains [25]. For other group III HKs it was already shown that mutations in the conserved phosphateaccepting residues and partial deletion of the HAMP domains conferred fungicide resistance $[23,26]$. This stimulated our interest to investigate the involvement of the HisKA, HATPase_c and REC domains from CaNik1p in the fungicide activity, as they are conserved in all HKs.

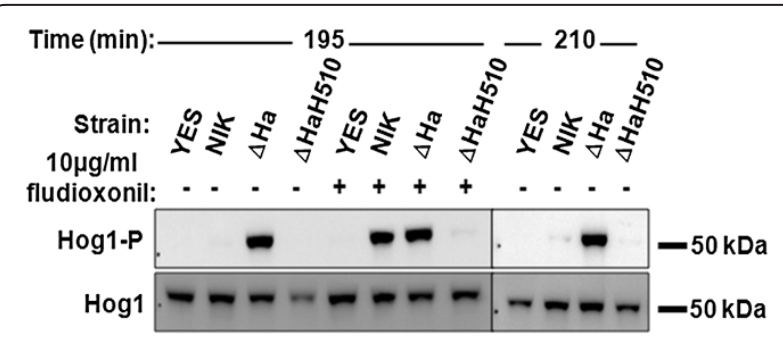

Figure 5 The MAPK Hog1p was constitutively phosphorylated after expression of CaNIK1 $\triangle H A M P$ in the strain $\triangle \mathrm{Ha}$.

Phosphorylation of Hog1p (upper panel, Hog1-P) in the strains YES, $\mathrm{NIK}, \Delta \mathrm{Ha}$ and $\Delta \mathrm{HaH} 510$ was detected after cultivation of the strains in SG-ura for 195 and 210 min. Fludioxonil was added after $180 \mathrm{~min}$ at a final concentration of $10 \mu \mathrm{g} / \mathrm{ml}$. The presence of Hog1p (lower panel, Hog1) was confirmed in all strains. Hog $1 p$ appears at approximately $50 \mathrm{kDa}$.
To prevent the primary phosphorylation of the histidine residue and the subsequent His-Asp phosphate-transfer from the HisKA to the REC domains, respectively, the point mutations H510Q and D924N were introduced. The N627D mutation was supposed to inactivate the ATP binding site. The complete resistance of the strains H510 and D924 and the reduced susceptibility of the strain N627 in comparison to the strain NIK clearly showed that the functionalities of the above mentioned domains were essential for the susceptibility of the transformed yeast to the tested fungicides. In agreement, similar patterns of Hog1p phosphorylation were obtained after treating the different $S$. cerevisiae transformants with fludioxonil. Phosphorylation of Hog1p was totally abolished in the strains H510 and D924 and partially inhibited in the strain N627, while in all strains expressing genes with point mutations Hog1p was phosphorylated in response to osmotic stress, but was not phosphorylated without external stimuli. These results are in agreement with earlier reports of reduced antifungal susceptibilities of strains, which expressed other group III HKs carrying point mutations in the HisKA and REC domains $[26,27]$. However, the correlation between the functionality of conserved HisKA, REC and HATPase_c domains of CaNik1p and both the fungicidal sensitivity and phosphorylation of Hog1p after fungicidal treatment was not shown before. Altogether, we present clear evidences that the histidine kinase functionality of CaNik1p was essential for the fungicidal effect and that this effect correlated with the activation of the MAPK Hog1p after treatment with fungicides.

The yeast histidine kinase Sln1p (group VI histidine kinase) is a negative regulator of the MAPK Hog1p, as its inhibition leads to activation of the MAPK. However, for group III HKs different effects were reported: Dic1p, the group III HK from Cochliobolus heterostrophus, was described as a positive regulator of Hog1p [24], whereas DhNik1p from Dabaryomyces hansenii was identified as a negative regulator [23]. To the best of our knowledge the relationship between the activity of CaNik1p and Hog1p has not yet been investigated, but can now be indirectly deduced from our data. It is generally accepted that activation of Hoglp in the absence of osmotic stress results in growth inhibitory effects [46]. Previously we reported that the antifungal effects of fludioxonil, iprodione and ambruticin VS3 are dependent on the Ssk1 - Pbs2 Hog1p branch of the osmotic stress response pathway [25], so that a prerequisite for phosphorylation of Hoglp is the non-phosphorylated form of the response regulator Ssk1p [47]. It was even reported that the presence of phosphorylated Ssk1p prevented the activation of the MAP3K Ssk2p from unphosphorylated Ssk1p [48]. Ssk1p receives phosphate groups indirectly from HKs via the histidine transfer protein Ypd1p. Our results indicate that this phosphorylation is inhibited only in strains which are exposed to 
osmotic stress or which express the wild-type CaNIK1 variants and are treated with fungicides. In strains expressing mutated non-functional CaNIK1 phosphorylation of Ssk1 was not inhibited. This conclusion is in agreement with [23] who showed that fludioxonil treatment of $S$. cerevisiae expressing the group III DhNik1p decreased the phosphate transfer to a response regulator even in the presence of the endogenous, active HK Sln1.

Group III HKs are characterized by an amino acid repeat domain with five to six amino acid repeats, in each of which a single HAMP domain was identified previously, but which are now known to comprise concatenated pairs of HAMP domains $[25,32,33]$. The function of these domains is not yet clear, even though involvement in fungicide susceptibility and in osmosensing were suggested $[19,23,25,37]$. Previous heterologous expression of truncated proteins, in which several HAMP domains were deleted from group III HKs, i.e. from CaNik1p [25] and DhNik1p from D. hansenii [37], was not reported to result in inhibition of growth of the respective $S$. cerevisiae transformants. Whereas in the previous reports only selected HAMP domains were deleted, here we deleted all HAMP domains from CaNik1p (CaNik1p $\triangle$ HAMP) and observed that the synthesis of this truncated protein in the transformed S. cerevisiae strain was associated with severe growth inhibition. This phenotype could be reversed by additional point mutation in the histidine phosphorylation site of the HisKA domain (H510) or by the expression of CaNIK1 $\triangle H A M P$ in single gene deletion mutants of the response regulator SSK1 or of one of the components of the Hog1 module namely the MAP2K PBS2 and the MAPK HOG1. This proved that the inhibition of growth of the transformant upon expression of CaNIK1 1 HAMP was dependent on the functionality of both the histidine kinase activity of CaNik1p and the functionality of the Ssk1 Pbs2 - Hog1 branch of the HOG pathway. We could also unambiguously show that the inhibited growth of the strain $\triangle \mathrm{Ha}$ expressing CaNIK1 $\triangle H A M P$ correlated with the constitutive phosphorylation of Hoglp without any external stimulation, which was also abolished after mutation of the conserved phosphate-accepting histidine residue (CaNIK1 1 HAMP(H510Q)). By analogy to the previous discussion this leads to the conclusion that the expression of CaNIK1 1 HAMP decreased the phosphate transfer activity to Ssk1, whereas the presence of the mutant CaNik1p $\triangle \mathrm{HAMP}(\mathrm{H} 510 \mathrm{Q})$, which cannot be phosphorylated on the conserved histidine residue, did not affect the endogenous phosphorylation state of the Ssk1p. Thus, in summary, deletion of all HAMP domains had the same effect on the phosphate transfer activity to Ssk1p as treatment with fungicides. Additionally, the presence of mutated proteins, which are assumed not to possess histidine kinase activity and thus are not phosphorylated on either histidine in the HisKA domain or on aspartate in the REC domain, did not inhibit growth of the transformants and did not activate the Hog1p MAPK module. As a consequence of these results, it seems to be unlikely that in the transformed S. cerevisiae strains the histidine kinase activity of CaNik1p was inhibited by fungicide treatment, because inhibition of the kinase activity will lead to an enrichment of the non-phosphorylated form of the protein, similar to the protein variants carrying point mutations. The mutated proteins, however, did not influence growth whereas fungicide treatment did. Thus, our results support a model, in which the wild-type CaNik1p protein is not phosphorylated without external stimuli, and Ssk1p is kept in a phosphorylated form via indirect phosphate transfer from Sln1p. Upon deletion of all HAMP domains from CaNik1p or fungicide treatment CaNik1p is phosphorylated and this form prevents phosphate transfer to Ssk1p (Figure 6).

However, this model is based on the assumption that the phosphorylation state of the endogenous histidine kinase $\operatorname{Snn} 1 \mathrm{p}$ is not changed by the presence of CaNik1p, since $\operatorname{Sln} 1 p$ is a transmembrane protein that undergoes autophosphorylation in the absence of osmotic stress and CaNik1p is a cystosolic protein. Thus, we expected that CaNik1p does not interfere with the autophosphorylation of the transmembrane protein $\operatorname{Sln} 1 \mathrm{p}$ but with the phosphate transfer from $\operatorname{Sin} 1 \mathrm{p}$ to Ypd1p. But it may also be hypothesized that when CaNik1p is phosphorylated, either following fungicide exposure or due to the absence of regulatory HAMP domains, Sln1p is no longer phosphorylated leading to the accumulation of non-phosphorylated forms of Ypd1p and Ssk1p and, hence, to the activation of Hog1p.

Thus, CaNik1p has to be considered as a positive regulator of Hog1p activity, similar to Dic1p from $C$. heterostrophus and contrary to DhNik1p and Sln1p. This is in agreement with the earlier results that CaNik1p cannot reverse the lethal phenotype of Sln1 deletion in S. cerevisiae whereas DhNik1p can. However, the mechanism leading to the reduced overall phosphate transfer activity to the response regulator remains to be investigated. As long as no protein structures are available from group III histidine kinases, one cannot exclude that point mutations and protein truncation have severe effects on the protein structures.

The constructed mutated versions of CaNIK1 could not be re-integrated in the available CaNIK1 homozygous deletion mutants of Candida albicans [8-18] as these mutants were constructed with the widely used URA blaster method and, thus, are prototrophic for uracil. Consequently direct transformation with the pYES2 vectors that harbor the mutated variants of the CaNIK1 was not possible as the vector contains URA3 


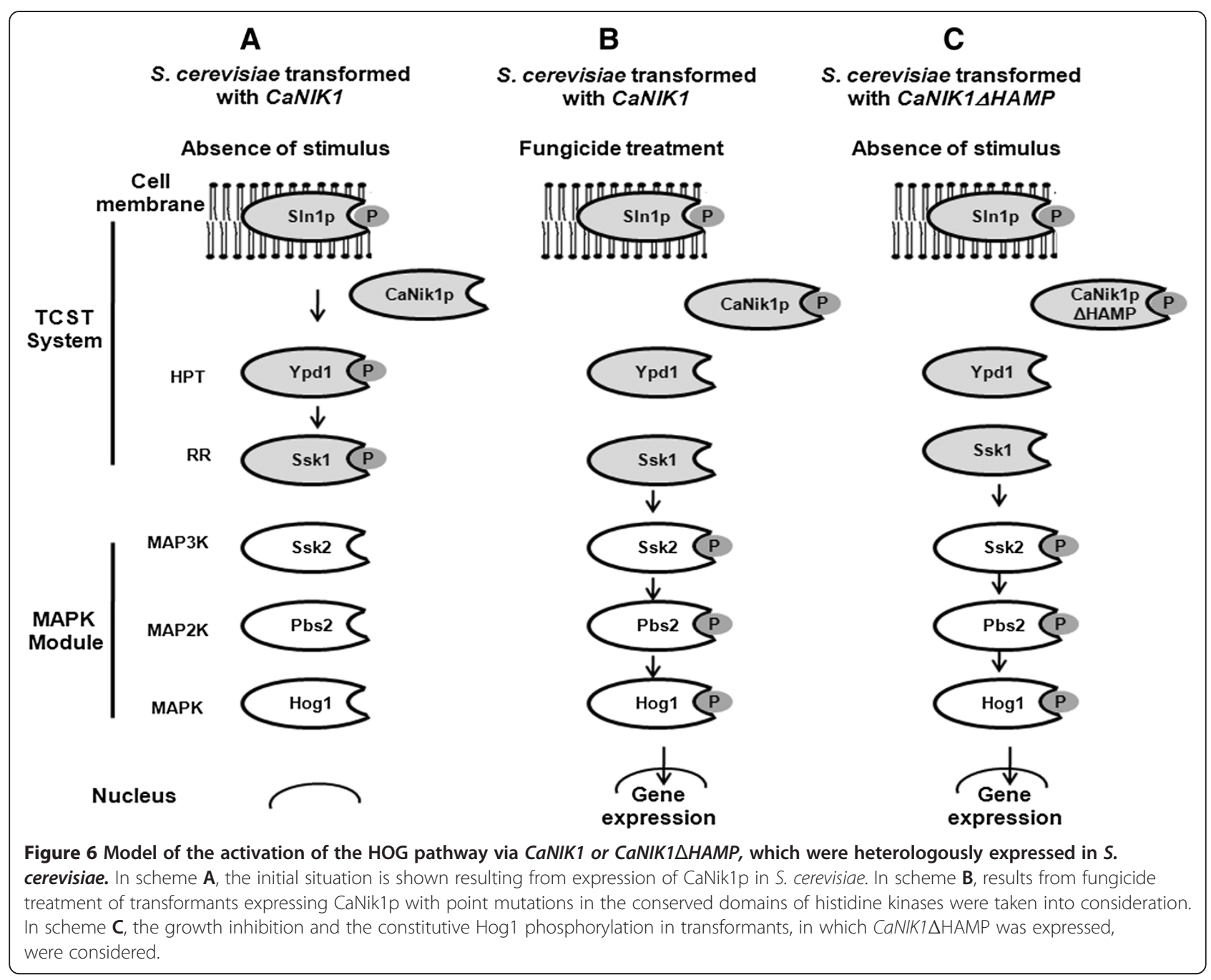

as a selection marker. Therefore a new CaNIK1 homozygous deletion mutant has to be constructed using for example the SAT1 flipper cassette that makes use of nourseothricin as an antibiotic selection marker. This will allow reintegration of the CaNIK1-mutated variants from this study in such mutant.

\section{Conclusion}

Our results show that functional HisKA, HATPase_c and REC domains of CaNik1p are essential for the antifungal activity of the selected agents activating the HOG pathway. Moreover, the expression of CaNIK1 LHAMP in transformed S. cerevisiae was associated with growth inhibition via constitutive phosphorylation of the MAPK Hog1p. In S. cerevisiae transformed with CaNIK1, growth inhibition resulting from treatment with the selected antifungals or from deletion of all HAMP domains from the protein required both a functional histidine kinase CaNik1p and an intact HOG pathway.

\section{Additional file}

Additional file 1: Expression of CaNIK1 $\triangle H A M P$ in the strain $\triangle \mathrm{Ha}$ was confirmed after 180 min cultivation in SG-ura. The strains NIK, $\triangle \mathrm{Ha}$ and $\triangle \mathrm{HaH} 510$ were cultivated in SG-ura for $180 \mathrm{~min}$ before the expression of CaNIK1, CaNIK1 $\triangle$ HAMP and CaNIK1 $\triangle H A M P$ (H510Q), respectively, was detected in the protein extracts via Western Blot using an anti-Flag antibody. The bands of CaNik1p, CaNik1p $\triangle$ HAMP and CaNik1p $\triangle$ HAMP(H510Q) appeared at 121,75 and $75 \mathrm{kDa}$ respectively. The strain YES with the empty vector was used as control.

\section{Abbreviations}

HK: Histidine kinase; HOG: High osmolarity glycerol; HPT: Histidine phosphotransfer protein; HRP: Horseradish Peroxidase; MAPK: Mitogenactivated protein kinase; MAP2K: Mitogen-activated protein kinase kinase; MAP3K: Mitogen-activated protein kinase kinase kinase; REC: Receiver domain; RR: Response regulator protein; TCST: Two component signal transduction; SD: Synthetic medium containing dextrose as carbon source; SG: Synthetic medium containing galactose and raffinose as carbon source; Ura: Uracil.

Competing interests

The authors declare that they have no competing interests. 


\section{Authors' contributions}

MEl-M planned and performed all experiments, presented the results and prepared the manuscript. MMB gave advice for the genetic manipulations, discussed results and contributed to manuscript preparation. UB devised and supervised the whole project, discussed results and prepared the final version of the manuscript. All authors read and approved the final manuscript.

\section{Acknowledgement}

We thank K. Gerth, H. Steinmetz, R. Jansen (all Research group Microbial Drugs (MWIS) of the HZI, Braunschweig) for providing us with ambruticin VS3, P. P. Müller (RDIF, HZI, Braunschweig) for frequent fruitful discussions, and $\mathrm{V}$. Wray ( $\mathrm{HZI}$, Braunschweig) for careful correction of the manuscript. This study was financially supported by a DAAD scholarship (M. El-M.) and by the Graduate School of the $\mathrm{HZI}$, Braunschweig. MMB was supported by a fellowship from the Alexander von Humboldt Foundation.

\section{Author details}

${ }^{1}$ AG Biological Systems Analysis, Helmholtz Centre for Infection Research (HZI), Inhoffenstr. 7, 38124 Braunschweig, Germany. ${ }^{2}$ Present address: Department of Microbiology and Immunology, Faculty of Pharmacy, Mansoura University, Mansoura, Egypt. ${ }^{3}$ Therapeutic Chemistry Department, Immunology and Infectious Diseases Research Group, the National Research Center, Cairo, Egypt. ${ }^{4}$ Present address: Research Group Biomarkers for Infectious Diseases, Institute for Experimental Infection Research, TWINCORE, Centre for Experimental and Clinical Infection Research, Feodor-Lynen-Str. 7 30625 Hannover, Germany.

Received: 16 May 2013 Accepted: 6 September 2013 Published: 17 September 2013

\section{References}

1. Roeder A, Kirschning CJ, Rupec RA, Schaller M, Weindl G, Korting HC: Tolllike receptors as key mediators in innate antifungal immunity. Med Mycol 2004, 42:485-498.

2. Miceli MH, Diaz JA, Lee SA: Emerging opportunistic yeast infections. Lancet Infect Dis 2011, 11:142-151.

3. Ruhnke M: Epidemiology of Candida albicans infections and role of nonCandida-albicans yeasts. Curr Drug Targets 2006, 7:495-504

4. Horn DL, Neofytos D, Anaissie EJ, Fishman JA, Steinbach WJ, Olyaei AJ, Marr KA, Pfaller MA, Chang CH, Webster KM: Epidemiology and outcomes of candidemia in 2019 patients: data from the prospective antifungal therapy alliance registry. Clin Infect Dis 2009, 48:1695-1703.

5. Vandeputte $P$, Ferrari $S$, Coste AT: Antifungal resistance and new strategies to control fungal infections. Int J Microbiol 2012, 2012:713687.

6. Myoken Y, Kyo T, Sugata T, Murayama SY, Mikami Y: Breakthrough fungemia caused by fluconazole-resistant Candida albicans with decreased susceptibility to voriconazole in patients with hematologic malignancies. Haematologica 2006, 91:287-288.

7. Chauhan N, Calderone R: Two-component signal transduction proteins as potential drug targets in medically important fungi. Infect Immun 2008, 76:4795-4803.

8. Yamada-Okabe T, Mio T, Ono N, Kashima Y, Matsui M, Arisawa M, YamadaOkabe $\mathrm{H}$ : Roles of three histidine kinase genes in hyphal development and virulence of the pathogenic fungus Candida albicans. J Bacteriol 1999, 181:7243-7247.

9. Catlett NL, Yoder OC, Turgeon BG: Whole-genome analysis of twocomponent signal transduction genes in fungal pathogens. Eukaryot Cell 2003, 2:1151-1161.

10. Nemecek JC, Wuthrich M, Klein BS: Global control of dimorphism and virulence in fungi. Science 2006, 312:583-588.

11. Kruppa M, Calderone R: Two-component signal transduction in human fungal pathogens. FEMS Yeast Res 2006, 6:149-159.

12. Desai C, Mavrianos J, Chauhan N: Candida albicans SRR1, a putative twocomponent response regulator gene, is required for stress adaptation, morphogenesis, and virulence. Eukaryot Cell 2011, 10:1370-1374.

13. Bahn YS: Master and commander in fungal pathogens: the twocomponent system and the HOG signaling pathway. Eukaryot Cell 2008, 7:2017-2036.

14. Maeda T, Wurgler-Murphy SM, Saito H: A two-component system that regulates an osmosensing MAP kinase cascade in yeast. Nature 1994 , 369:242-245.
15. Appleby JL, Parkinson JS, Bourret RB: Signal transduction via the multi-step phosphorelay: not necessarily a road less traveled. Cell 1996, 86:845-848.

16. Nagahashi S, Mio T, Ono N, Yamada-Okabe T, Arisawa M, Bussey H, YamadaOkabe H: Isolation of CaSLN1 and CaNIK1, the genes for osmosensing histidine kinase homologues, from the pathogenic fungus Candida albicans. Microbiology 1998, 144(Pt 2):425-432

17. Srikantha T, Tsai L, Daniels K, Enger L, Highley K, Soll DR: The twocomponent hybrid kinase regulator CaNIK1 of Candida albicans. Microbiology 1998, 144(Pt 10):2715-2729.

18. Alex LA, Korch C, Selitrennikoff CP, Simon MI: COS1, a two-component histidine kinase that is involved in hyphal development in the opportunistic pathogen Candida albicans. Proc Natl Acad Sci USA 1998, 95:7069-7073.

19. Ochiai N, Fujimura M, Motoyama T, Ichiishi A, Usami R, Horikoshi K, Yamaguchi I: Characterization of mutations in the two-component histidine kinase gene that confer fludioxonil resistance and osmotic sensitivity in the os-1 mutants of Neurospora crassa. Pest Manag Sci 2001, 57:437-442

20. Ochiai N, Fujimura M, Oshima M, Motoyama T, Ichiishi A, Yamada-Okabe $H_{\text {, }}$ Yamaguchi I: Effects of iprodione and fludioxonil on glycerol synthesis and hyphal development in Candida albicans. Biosci Biotechnol Biochem 2002, 66:2209-2215.

21. Motoyama T, Kadokura K, Ohira T, Ichiishi A, Fujimura M, Yamaguchi I, Kudo T: A two-component histidine kinase of the rice blast fungus is involved in osmotic stress response and fungicide action. Fungal Genet Biol 2005, 42:200-212.

22. Knauth $\mathrm{P}$, Reichenbach $\mathrm{H}$ : On the mechanism of action of the myxobacterial fungicide ambruticin. J Antibiot (Tokyo) 2000, 53:1182-1190.

23. Furukawa K, Randhawa A, Kaur H, Mondal AK, Hohmann S: Fungal fludioxonil sensitivity is diminished by a constitutively active form of the group III histidine kinase. FEBS Lett 2012, 586:2417-2422.

24. Yoshimi A, Kojima K, Takano Y, Tanaka C: Group III histidine kinase is a positive regulator of Hog1-type mitogen-activated protein kinase in filamentous fungi. Eukaryot Cell 2005, 4:1820-1828

25. Buschart A, Gremmer K, El-Mowafy M, van den Heuvel J, Mueller PP, Bilitewski U: A novel functional assay for fungal histidine kinases group III reveals the role of HAMP domains for fungicide sensitivity. J Biotechnol 2012, 157:268-277.

26. Motoyama T, Ohira T, Kadokura K, Ichiishi A, Fujimura M, Yamaguchi I, Kudo $\mathrm{T}$ : An Os-1 family histidine kinase from a filamentous fungus confers fungicide-sensitivity to yeast. Curr Genet 2005, 47:298-306.

27. Vetcher L, Menzella HG, Kudo T, Motoyama T, Katz L: The antifungal polyketide ambruticin targets the HOG pathway. Antimicrob Agents Chemother 2007, 51:3734-3736.

28. Dongo A, Bataille-Simoneau N, Campion C, Guillemette T, Hamon B, lacomiVasilescu B, Katz L, Simoneau P: The group III two-component histidine kinase of filamentous fungi is involved in the fungicidal activity of the bacterial polyketide ambruticin. Appl Environ Microbiol 2009, 75:127-134.

29. Tao W, Malone CL, Ault AD, Deschenes RJ, Fassler JS: A cytoplasmic coiledcoil domain is required for histidine kinase activity of the yeast osmosensor, SLN1. Mol Microbiol 2002, 43:459-473.

30. Dutta $R$, Inouye M: Reverse phosphotransfer from OmpR to EnvZ in a kinase-/phosphatase + mutant of EnvZ (EnvZ.N347D), a bifunctional signal transducer of Escherichia coli. J Biol Chem 1996, 271:1424-1429.

31. Aravind L, Ponting CP: The cytoplasmic helical linker domain of receptor histidine kinase and methyl-accepting proteins is common to many prokaryotic signalling proteins. FEMS Microbiol Lett 1999, 176:111-116.

32. Dunin-Horkawicz S, Lupas AN: Comprehensive analysis of HAMP domains: implications for transmembrane signal transduction. J Mol Biol 2010, 397:1156-1174.

33. Airola MV, Watts KJ, Bilwes AM, Crane BR: Structure of concatenated HAMP domains provides a mechanism for signal transduction. Structure 2010, 18:436-448

34. Appleman JA, Stewart V: Mutational analysis of a conserved signaltransducing element: the HAMP linker of the Escherichia coli nitrate sensor NarX. J Bacteriol 2003, 185:89-97.

35. Hulko M, Berndt F, Gruber M, Linder JU, Truffault V Schultz A Martin J, Schultz JE, Lupas AN, Coles M: The HAMP domain structure implies helix rotation in transmembrane signaling. Cell 2006, 126:929-940.

36. Swain KE, Falke JJ: Structure of the conserved HAMP domain in an intact, membrane-bound chemoreceptor: a disulfide mapping study. Biochemistry 2007, 46:13684-13695. 
37. Meena $\mathrm{N}$, Kaur $\mathrm{H}$, Mondal AK: Interactions among HAMP domain repeats act as an osmosensing molecular switch in group III hybrid histidine kinases from fungi. J Biol Chem 2010, 285:12121-12132.

38. Guarente L, Mason T: Heme regulates transcription of the CYC1 gene of S. cerevisiae via an upstream activation site. Cell 1983, 32:1279-1286.

39. Brachmann CB, Davies A, Cost GJ, Caputo E, Li J, Hieter P, Boeke JD: Designer deletion strains derived from Saccharomyces cerevisiae S288C: a useful set of strains and plasmids for PCR-mediated gene disruption and other applications. Yeast 1998, 14:115-132.

40. Amberg DC, Burke D, Strathern JN: Methods in Yeast Genetics: A Cold Spring Harbor Laboratory Manual. NY: Cold Spring Harbor Laboratory Press; 2005.

41. Hofle G, Steinmetz H, Gerth K, Reichenbach H: Antibiotics from gliding bacteria, XLIV. Ambruticins VS: New members of the antifungal ambruticin family from Sorangium cellulosum. Liebigs Ann Chem 1991, 1991:941-945.

42. Gustin MC, Albertyn J, Alexander M, Davenport K: MAP kinase pathways in the yeast Saccharomyces cerevisiae. Microbiol Mol Biol Rev 1998, 62:1264-1300.

43. Panadero J, Pallotti C, Rodriguez-Vargas S, Randez-Gil F, Prieto JA: A downshift in temperature activates the high osmolarity glycerol (HOG) pathway, which determines freeze tolerance in Saccharomyces cerevisiae. J Biol Chem 2006, 281:4638-4645.

44. Smith PK, Krohn RI, Hermanson GT, Mallia AK, Gartner FH, Provenzano MD, Fujimoto EK, Goeke NM, Olson BJ, Klenk DC: Measurement of protein using bicinchoninic acid. Anal Biochem 1985, 150:76-85.

45. Wurgler-Murphy SM, Maeda T, Witten EA, Saito H: Regulation of the Saccharomyces cerevisiae HOG1 mitogen-activated protein kinase by the PTP2 and PTP3 protein tyrosine phosphatases. Mol Cell Biol 1997, 17:1289-1297.

46. Posas F, Wurgler-Murphy SM, Maeda T, Witten EA, Thai TC, Saito H: Yeast HOG1 MAP kinase cascade is regulated by a multistep phosphorelay mechanism in the SLN1-YPD1-SSK1 "two-component" osmosensor. Cell 1996, 86:865-875.

47. Posas F, Saito H: Activation of the yeast SSK2 MAP kinase kinase kinase by the SSK1 two-component response regulator. EMBO J 1998, 17:1385-1394.

48. Horie T, Tatebayashi K, Yamada R, Saito H: Phosphorylated Ssk1 prevents unphosphorylated Ssk1 from activating the Ssk2 mitogen-activated protein kinase kinase kinase in the yeast high-osmolarity glycerol osmoregulatory pathway. Mol Cell Biol 2008, 28:5172-5183.

49. Winzeler EA, Shoemaker DD, Astromoff A, Liang H, Anderson K, Andre B, Bangham R, Benito R, Boeke JD, Bussey H: Functional characterization of the $S$. cerevisiae genome by gene deletion and parallel analysis. Science 1999, 285:901-906.

doi:10.1186/1471-2180-13-209

Cite this article as: El-Mowafy et al:: Deletion of the HAMP domains from the histidine kinase CaNik1p of Candida albicans or treatment with fungicides activates the MAP kinase Hog1p in S. cerevisiae transformants. BMC Microbiology 2013 13:209.

\section{Submit your next manuscript to BioMed Central and take full advantage of:}

- Convenient online submission

- Thorough peer review

- No space constraints or color figure charges

- Immediate publication on acceptance

- Inclusion in PubMed, CAS, Scopus and Google Scholar

- Research which is freely available for redistribution

Submit your manuscript at www.biomedcentral.com/submit
Ciomed Central 